\title{
IMPACTO DEL PROCESO DE ACREDITACIÓN DE CARRERAS EN EL MEJORAMIENTO DE LA GESTIÓN ACADÉMICA
}

\section{Antecedentes y justificación del estudio}

Dar fe pública de la calidad de la educación superior se ha transformado en un imperativo para los gobiernos de los países que aspiran al desarrollo. Ya sea por resguardar el derecho de la ciudadanía a una formación de excelencia, o por asegurar la calidad del capital humano, y con ello su competitividad, prácticamente ningún país ha quedado al margen de este proceso. Internacionalmente, los sistemas de aseguramiento de la calidad de la educación están en proceso de maduración. Ciertamente, algunos países como Estados Unidos, Inglaterra, o Chile (en Latinoamérica) llevan la delantera en los principales desafíos del sistema, entre los cuales destacan asegurar públicamente la calidad de las instituciones de educación superior (IES), mantener un sistema transparente y autónomo y contribuir eficientemente a la decisión profesional de los postulantes a la educación superior. 


\section{Cuadro resumen}

Desarrollo del contexto de la educación superior con los antecedentes de la definición de la política de Aseguramiento de Calidad de la Educación Superior, que se implementa en la actualidad a través de la Comisión Nacional de Acreditación de Pregrado (CNAP) y de la futura Comisión Nacional de Acreditación (CNA).

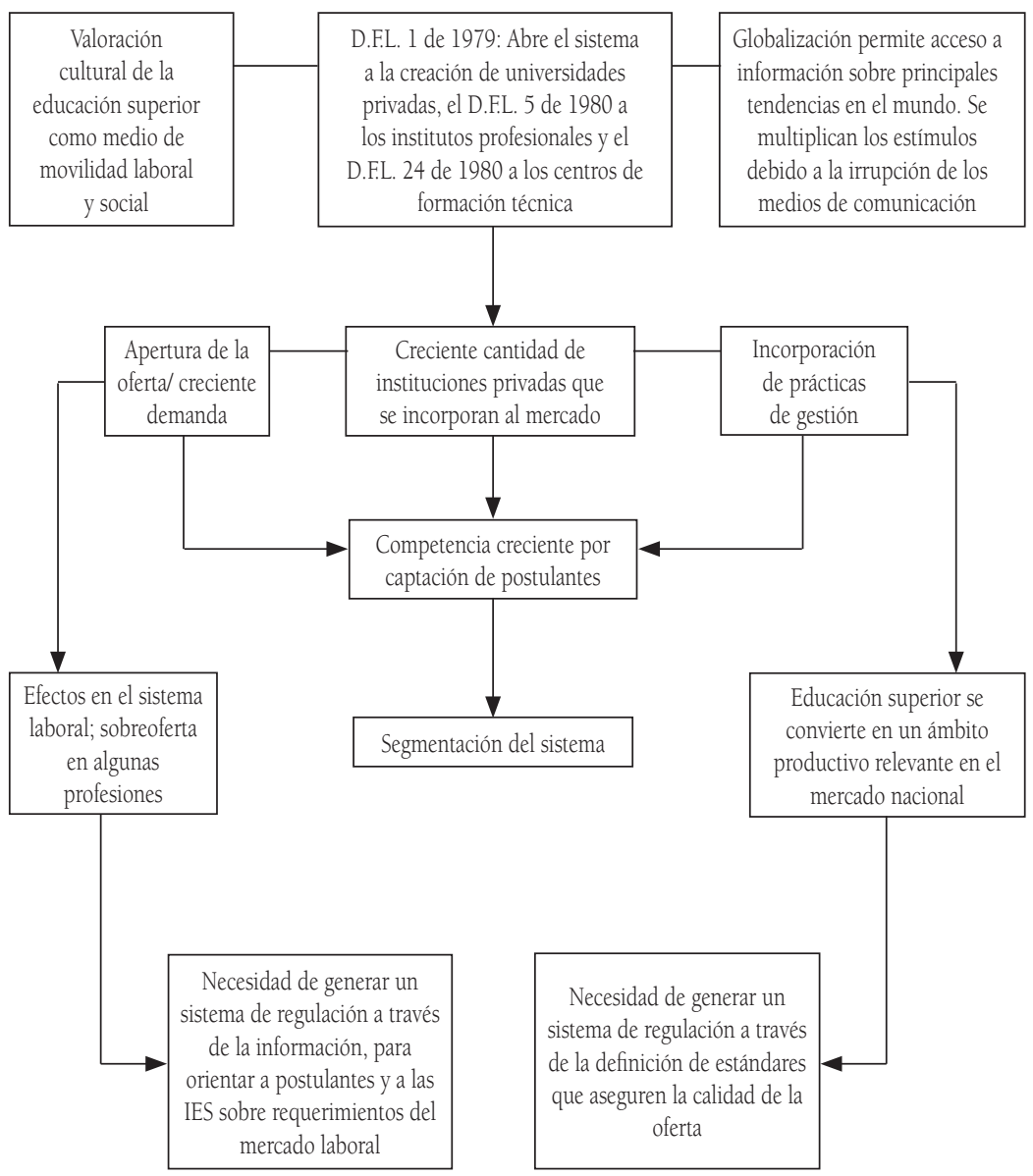

En nuestro país, durante la gestión de los gobiernos de la Concertación de Partidos por la Democraciaํ (1990 a la fecha), la

1 Coalición que gobierna Chile desde el retorno de la democracia. 
información pública, los medios de comunicación y las nuevas políticas educativas -caracterizadas por un alto componente participativohan puesto en evidencia la situación deficiente de la educación chilena y, asimismo, la preocupación pública por todos sus niveles (prebásica, básica, media y superior). Habitualmente, la ciudadanía ha centrado su atención en la educación primaria y secundaria, en tanto la obligatoriedad de dichos niveles es responsabilidad del Estado, pero también porque la educación terciaria ha sido, desde el gobierno militar hasta hoy, un ámbito privado en términos de información y desregulado, con características que lo distancian del contexto académico tradicional y lo acercan a la dinámica propia de un sistema de mercado (incluso las instituciones públicas). En este ámbito, la información es asimétrica y la selección del producto varía irregularmente según diversos criterios diferenciadores no necesariamente académicos.

Es posible apreciar que, independientemente de la modalidad, la regulación es un fenómeno que se ha incorporado con fuerza en la última década al ámbito de la educación superior y esto ha traído implicancias para los sectores público y privado, en tanto se han exigido permanentes acciones de accountability, ya sea respecto de la gestión con recursos estatales o aquellos que provienen de aranceles pagados por los estudiantes; en otras palabras, ambos tipos de instituciones han debido implementar sistemas de monitoreo, evaluación y publicación de resultados. "Esencialmente, la evaluación institucional es un componente de la gestión. En principio, cualquier organización o empresa, con o sin fines de lucro, productiva o de servicios, pública o privada, necesita conocer, de manera sistemática y objetivada, esto es con el respaldo de datos duros (cifras y hechos) y blandos (opinión de actores internos y externos pertinentes), tanto la situación de su interior como del entorno". (Silva, Ramírez y Atria, 2002: 28).

A partir de la definición de estrategias de política pública para fortalecer el nivel terciario, en 1997 se generó en Chile un programa especialmente destinado a mejorar la calidad y equidad de la educación 
superior: el Programa de Mejoramiento de la Calidad y Equidad de la Educación Superior (MECESUP). En 1999, para fortalecer las acciones tendientes a tal objetivo, se crea la Comisión Nacional de Acreditación de Pregrado (CNAP) $)^{2}$, encargada, por una parte, de desarrollar procesos experimentales de acreditación -inicialmente de carreras y, luego también, de instituciones de educación superior-y, por otra, de sentar las bases de un Sistema Nacional de Aseguramiento de la Calidad.

El proceso de acreditación de carreras y programas define tres etapas constitutivas a las que deben someterse las unidades académicas: Informe de autoevaluación, Visita e Informe de pares expertos y, finalmente, deliberación y dictamen de la Comisión. El objetivo principal es, "Fomentar y dar garantía pública de la calidad de las carreras a través de ejercicios sistemáticos de evaluación" (CNAP, 2006). El interés particular del presente análisis es conocer el impacto de los procesos de acreditación-específicamente del proceso de autoevaluación- en el mejoramiento de la gestión académica y, en particular, en la generación o profundización de una cultura de autorregulación en las carreras.

La CNAP entiende por "carrera" un programa de formación o propuesta curricular impartido por una universidad, instituto profesional o centro de formación técnica; sin embargo, para efectos de este estudio, sólo se contemplarán casos de universidades. Asimismo, la expresión "unidad(es) académica(s)" (UA) se usará como sinónimo de "carrera(s)" o "programa(s) de pregrado".

Con el fin de facilitar el proceso de autoevaluación, centro medular de la acreditación, la CNAP definió nueve criterios que permiten medir la eficacia externa e interna de cada carrera o programa. Autoevaluar consiste en verificar la correspondencia de los perfiles profesionales con estos nueve criterios: Propósitos, Integridad, Estructura organizacional, administrativa y financiera, Estructura curricular, Recursos humanos, Efectividad del proceso enseñanza aprendizaje, Resultados del proceso

2 Actualmente, sólo Comisión Nacional de Acreditación. 
de formación, Infraestructura, apoyo técnico y recursos para la enseñanza, y Vinculación con el medio.

El espíritu de este proceso es promover la implementación de sistemas de autorregulación de la calidad en las UA, propendiendo, de esta forma, al desarrollo de una cultura de calidad permanente e instalada en cada sistema educativo y no, como podría pensarse, en entes externos (control). Por eso se ha definido un carácter cíclico del proceso en el que, incluso aquellas carreras que se acreditan por el máximo de años ${ }^{3}$, deberán, al cabo de este período, someterse nuevamente al mismo procedimiento. Esto implicaría una verdadera transformación del sistema de educación superior chileno: el paso hacia una cultura de autorregulación se traduciría en un verdadero cambio estructural, tanto en las carreras como en la mentalidad de oferentes y demandantes, o sea, en la relación y dinámica que caracteriza el mercado. Se esperaría que directivos, docentes, alumnos y funcionarios en general contribuyeran permanentemente al mejoramiento continuo del servicio, a la implementación exitosa de innovaciones y, en definitiva, al paso hacia una formación de mejor calidad, tanto en el sector público como privado.

El cambio paradigmático, tras la difusión de una cultura de autorregulación y de los sistemas de aseguramiento a nivel internacional, supone, entre otras cosas, contar con UA participativas, flexibles y transparentes. Participativas porque toda la comunidad académica es la que construye el proyecto educativo y, por ende, debe estar convencida e involucrada en el proceso; flexible, en tanto deben planificarse políticas y procesos que permitan responder a los cambios permanentes del contexto, y transparentes, ya que la optimización de sus resultados depende de la socialización de toma de decisiones y resultados para su mejoramiento.

Recientemente ha entrado en vigencia la Ley $20.129^{4}$ de Aseguramiento de la Calidad, la cual, ciertamente, cambiará las

3 Máximo siete y mínimo dos.

4 Publicada con fecha 17 de noviembre de 2006, MINEDUC. 
características del proceso. Actualmente, hay desinformación y una preocupación casi generalizada, porque una de sus principales características es la delegación de la función acreditadora de carreras en agencias privadas. Hasta hoy sólo una agencia ha sido autorizada por la CNAP y con carácter experimental.

\section{Referentes conceptuales}

A partir de una investigación realizada con académicos del Reino Unido respecto de la acreditación, se pudo concluir que si bien reconocen la necesidad de implementar un sistema regulatorio que permita formalizar y estandarizar los estudios y títulos superiores, perciben que el sistema aún tiene un alto costo de oportunidad para ellos, ya que lo ven como un sistema burocrático cuyos resultados, en términos de mejorar la calidad, sólo pueden observarse aun en un nivel superficial. A esto se suma la percepción de la acreditación como una forma de control externo a los departamentos y facultades, lo que amenaza la autonomía profesional de los cuerpos docentes. Resulta paradójico que uno de los efectos de esta sensación de amenaza externa es que, según los docentes entrevistados, los equipos de trabajo han mejorado sus relaciones por la necesidad de trabajar juntos para enfrentar un desafío común (Hoecht, 2006).

Otra investigación, realizada en países asiáticos, destaca algunos criterios de análisis para conocer y comparar los sistemas de acreditación. Entre ellos destacan las características del mercado universitario y los objetivos de los gobiernos al implementar un sistema de regulación; por ejemplo, señala la diferencia entre Hong-Kong y Singapur. El primero ha demostrado resultados de mayor impacto en los procesos pedagógicos que el segundo. Entre otros factores, porque el sistema de acreditación de Hong-Kong ha basado su diseño e implementación en los procesos, resultados e investigaciones de la experiencia implementada en el Reino Unido. Por su parte, el sistema universitario de Singapur es pequeño y heterogéneo (sólo cuenta con dos universidades de proyectos pedagógicos divergentes) y la generación de estándares comparativos es prácticamente imposible; 
por lo tanto, las estrategias de aseguramiento de la calidad se concentran en promover la autoevaluación y la autorregulación de cada institución.

Puede observarse que una política de acreditación en un país como Singapur persigue objetivos muy distintos a la política de Hong-Kong, que cuenta con menor cantidad de recursos y mayor cantidad de oferta institucional. Mientras en este último país el sistema de acreditación busca mejorar la productividad y el desarrollo de la universidad, para justificar la inversión de recursos públicos y asegurar una competencia transparente, en Singapur el objetivo central es asegurar la competitividad de la nación en el mercado global (Mok, 2000).

Según González (2005), la creación de una cultura de calidad en el sistema y las instituciones de Latinoamérica ha sido también el impacto más importante, traduciéndose en mayores niveles de responsabilidad por parte de los actores. Esta situación de mejoramiento se ha traducido incluso en una modificación del concepto de autonomía universitaria por el de "autonomía responsable", que combina la libertad académica con la responsabilidad social. Como consecuencia, las universidades han perfeccionado sus procesos de planificación y coherencia entre el plano operativo y el estratégico, mediante la profesionalización de la gestión universitaria. Otros impactos de la acreditación que González visualiza en el medio universitario latinoamericano son el diseño de reformas curriculares, reflexiones pedagógicas, alianzas de cooperación internacional y el avance hacia sistemas de información cada vez más transparentes.

En Chile, según Silva y Letelier (2003), el bagaje empírico acumulado ha contribuido a desarrollar una cultura de autoevaluación que se ha traducido en algunas transformaciones, como nuevas estructuras organizacionales en las instituciones para apoyar los procesos, aprendizaje en el diseño de procesos de autoevaluación y evaluación externa, capacitación de personal en el desempeño como pares evaluadores externos y en áreas de gestión, mejoramiento 
del conocimiento de las realidades internas de las instituciones, surgimiento de un campo profesional asociado al tema de la evaluación institucional e, incluso, la plena autonomía de algunas instituciones.

Otros impactos observables en nuestro país son aquellos que el organismo central ha implementado en los instrumentos y el proceso. En la medida en que se ha ido evaluando el proceso, así mismo ha evolucionado la valoración de la autoevaluación y la priorización de los criterios de calidad que hacen las instituciones. Iván Navarro, citando a Lemaitre (Navarro, 2006), identifica, para el caso chileno, tres etapas en la regulación de la calidad: "La regulación corporativa que ejercieron las propias instituciones de educación superior, a partir de la inicial hegemonía académica de la Universidad de Chile; la desregulación del sistema traducida en una reconfiguración de las universidades tradicionales y el expansionismo y diversificación del sistema, que lo llevó a pasar de las ocho universidades tradicionales a la desagregación en 300 instituciones en 1990, entre universidades tradicionales y derivadas, universidades privadas, institutos profesionales y centros de formación técnica; y la etapa de la regulación pública, compuesta a su vez por tres momentos: el del licenciamiento, ejercido por el Consejo Superior de Educación, creado por la LOCE (1990), y por la División de Educación Superior del MINEDUC; el de la acreditación voluntaria de carreras y programas iniciada con la creación de la CNAP (1999) y el de la acreditación institucional, centrada en la capacidad de autorregulación de las propias instituciones" (Navarro, 2006: 15) 5 .

El primer concepto que interesa desarrollar es el de regulación, en tanto ello nos da cuenta del grado de apertura de las UA: a mayor apertura a sistemas regulatorios, mayor disposición a involucrarse en el proceso de acreditación. En el contexto chileno se entiende que la regulación está dirigida a corregir deficiencias del sistema de mercado para satisfacer objetivos colectivos o de interés público. “...la

5 Iván Navarro Abarzúa ha sido miembro de la CNAP desde su creación, en 1999, hasta la fecha. 
palabra regulación se utiliza para designar a un cuerpo específico de normas jurídicas obligatorias, dirigido fundamentalmente a exigir o prohibir determinadas conductas, en que se estipulan sanciones ante su incumplimiento, y que es usualmente aplicada, monitoreada y fiscalizada por una entidad administrativa. Puede, también, ser entendida como una deliberada intervención del Estado en la economía y en la sociedad, en general, destinada a influenciar el comportamiento de la industria y la sociedad. Este concepto más amplio comprende al anterior, incluyendo, además, una amplia variedad de instrumentos o modos de influencia, como, por ejemplo, aquellos basados en incentivos económicos (tales como impuestos, subsidios, permisos transables, entre otros)" (Romero, 2005).

En la base de los sistemas de acreditación se encuentra la evaluación, una práctica que, a la luz de los avances de la gestión, ha evolucionado desde ser el resultado de una observación vertical con efectos de continuidad o desvinculación (término o cierre), hacia un cúmulo de observaciones provenientes desde los diversos actores involucrados en un proceso (en este caso académico) y conducente a sugerencias de mejora y desarrollo.

El procedimiento de evaluación puede aplicarse total o parcialmente a las instituciones, tal es el caso de la acreditación institucional o de programas, como señalan Silva, Ramírez y Atria (2002). Asimismo, puede responder a un propósito de desarrollo interno o externo, y es posible adoptar diversas modalidades de diseño y ejecución que se diferencian según la responsabilidad de quien conduce el proceso. Entre ellas destacan dos: la autoevaluación o autoestudio, a cargo de la propia institución o programa, y la evaluación externa, a cargo de una agencia externa (Silva, Ramírez y Atria, 2002).

La autoevaluación es un proceso en el que la institución se mide voluntariamente fundada en dos referentes: los estándares definidos externamente y la coherencia con su proyecto declarado. "Así, aunque este proceso surgió en el marco de la búsqueda de reconocimiento 
externo, bien gubernamental o de las agencias encargadas de acreditar la calidad, se ha ido constituyendo en un componente cada vez más relevante dentro de la cultura organizacional de las universidades. En este sentido, se ha ido incorporando dentro de los planes estratégicos como mecanismo para mantener niveles de calidad, acordes con los requerimientos de la sociedad del conocimiento" (ACAP, 2006).

Es importante destacar la diferencia entre lo que CNAP define como "autoevaluación" (AE) y lo que hemos denominado "autorregulación" (AR). Si bien prácticamente todas las definiciones de AR provienen de la economía, la cibernética o la biología, la AE es un procedimiento en el que deben realizarse tareas para verificar el cumplimiento de indicadores en un momento específico de la historia de la UA; la AR, en cambio, hace referencia a una condición instalada en la plataforma organizacional, que implica aspectos culturales, procedimientos, sistemas de información, estructura y procesos organizacionales permanentes y definidos para asegurar la optimización de sus procesos.

La AE puede ser enfrentada como una oportunidad de invertir recursos humanos, financieros y materiales, con el fin de implementar una cultura de AR que permanezca en el tiempo o, simplemente, como un procedimiento de toma de decisiones y destinación de gastos que permitan el cumplimiento de una tarea conducente a un sello de calidad. Así como la cultura, el clima en que se desenvuelven los procesos de aseguramiento de la calidad es sumamente relevante. La estrategia comunicacional que establecen las unidades coordinadoras en cada institución parece ser determinante en los procesos y resultados: los climas caracterizados por la cooperación, la confianza, el respeto, entre otros, son facilitadores de resultados propicios.

Las organizaciones educacionales se encuentran actualmente en una doble encrucijada: por una parte, como toda organización moderna, deben gestionar cambios que les permitan avanzar hacia la vertiginosa modernidad, respondiendo a los múltiples cambios y exigencias del contexto; por otra, deben asegurar la coherencia 
de dicho avance con el proyecto educativo que las ilumina. En este sentido, es importante agregar a este análisis bases conceptuales asociadas al desarrollo organizacional (DO), que según Darío Rodríguez es "un proceso de cambio planificado de la organización, para el que es necesario -en primer lugar-conocer la situación por la que atraviesa (la institución) y -luego- evaluar los resultados de los cambios propuestos e implementados" (Rodríguez, 1999).

Según Bennis, el DO es una respuesta al cambio, una compleja estrategia educacional que pretende cambiar las creencias, actitudes, valores y estructura de las organizaciones, de manera que se puedan adaptar mejor a los nuevos mercados, tecnologías y retos, y al vertiginoso ritmo del cambio mismo (French y Bell, 1996).

Con todo, es posible concluir que el proceso de DO de una institución nos permite conocer su capacidad de cambio planificado, su apuesta por acceder a estados superiores de desarrollo, involucrando el bienestar de las personas que la conforman, así como las expectativas de maximización de su productividad. Además, las características de desarrollo de una organización permiten conocer sus prácticas, sus capacidades, sus limitaciones, entre otros aspectos.

En palabras de Darío Rodríguez, "la organización es un sistema autopoiético de decisiones determinado estructuralmente que se encuentra en una relación permanente de acoplamiento estructural con su entorno (interno y externo) (...) El que sea un sistema autopoiético de decisiones significa que es una red de producción de decisiones que produce las decisiones que la forman. El que sea determinado estructuralmente quiere decir que todos los cambios que la organización experimenta son cambios posibles en su estructura". Agrega que el determinismo estructural se refiere a que en la estructura está definido el número de cambios posibles y que sólo en caso de que sometamos a la organización a ciertas "perturbaciones" es posible gatillar nuevas variaciones, lo que se traduciría en una nueva estructura y la definición de un nuevo número limitado de modificaciones. El autor menciona también la importancia de la 
adaptación al entorno, es decir, de la pertinencia de la organización a los cambios permanentes de su entorno, condición que, por una parte, asegura su supervivencia y, por otra, gatilla cambios internos. (Rodríguez, 2001)

De las palabras de Rodríguez podemos deducir que no basta con la definición de un sistema de regulación para la implementación de una cultura de calidad. Si efectivamente requerimos de las instituciones de educación superior (IES) un cambio permanente y una actitud de autorregulación, debemos visualizar sus oportunidades en la estructura y características del contexto interno, es decir, las implicancias de dicha estructura en las relaciones y conductas de las personas que conforman la institución y viceversa. En este sentido, podríamos asumir que no hay cambio posible si no parte por las personas que constituyen el sistema.

Sabemos, por teoría o por experiencias personales, que cualquier cambio provoca resistencias en las organizaciones, específicamente en grupos de interés que pueden sentirse amenazados. A esto Rodríguez responde que la estrategia más segura es planificar el cambio y que ello implica contar con adecuados sistemas de diagnóstico que permitan detectar con anticipación las resistencias. Asimismo, "la evaluación constante y la revisión de objetivos iniciales permiten un mayor ajuste entre la realidad y lo intentado" (Rodríguez, 2001). En lo que concierne a la acreditación, entonces, este desafío debe ser tomado en cuenta por la política pública, porque, para su implementación, deben visualizarse primero las oportunidades y amenazas del sistema nacional de IES. Éstas deben contar con un mapa claro que les permita generar estrategias para la difusión, socialización e implementación de un sistema de autorregulación. Si bien esto se traduce en aspectos operativos, presupuesto, destinación de personal, definición de procedimientos, entre otros insumos, también debe haber un convencimiento generalizado de los beneficios del nuevo sistema: "todo cambio efectivo, por tanto, es un cambio en las reglas y, al mismo tiempo, un cambio actitudinal" (Rodríguez, 2001) 
De esta forma llegamos a un segundo concepto de la teoría organizacional, importante para esta investigación: el de aprendizaje organizacional (AO). Éste involucra las relaciones que se establecen en la organización y que pueden ser vistas como mecanismos de reforzamiento entre variables.

Peter Senge, principal precursor moderno del concepto "aprendizaje organizacional", lo define como el resultado de lo que un grupo de personas puede llegar a realizar por su condición de grupo, es decir, lo que realizan colectivamente para producir el resultado deseado involucrando sus capacidades personales. Senge destaca que, en ciertos grupos, el resultado no es producto de las condiciones o la disposición inicial del grupo, sino, más bien, de sus interacciones y devenir (Senge, 2000)

Es decir, que el AO es un proceso mediante el cual las IES adquieren y crean conocimiento a través de las personas que la componen, sean éstas funcionarios o usuarios. Con ello, el conocimiento se vuelve institucional y permite a la organización adaptarse a las condiciones cambiantes de su entorno y, asimismo, transformarlo. Los procesos universitarios siempre están asociados a la generación de conocimiento, al aprendizaje y a otros objetivos de carácter educativo. Entonces, una tercera línea conceptual debe ser la que nos señale cómo gestionar dichos procesos.

Interesa conocer la capacidad de $\mathrm{AO}$ involucrado en la obtención de resultados en un proceso de acreditación de calidad de la educación, y cómo se asegura su permanencia y desarrollo en el mismo sistema educativo una vez superado el procedimiento. Para estos efectos, en la entrevista que se realizó a los coordinadores de las carreras se les solicitó que identificaran su "estado de aprendizaje" entre las siguientes categorías definidas por la investigadora:

a) Responde al procedimiento: todas aquellas IES que se someten al proceso de autoevaluación cumpliendo con las tareas en función del plazo y del logro de la acreditación.

b) Genera información: todas aquellas IES que, para el cumplimiento 
de la tarea, sistematizan y generan un sistema de información disponible públicamente para otros procesos.

c) Reingeniería: todas aquellas IES que, luego de someterse al proceso de autoevaluación, aprovechan la información para generar cambios estructurales, es decir, están dispuestas a invertir recursos en su desarrollo hacia mejores y permanentes niveles de calidad.

d) Autorregulación: todas aquellas IES que generan propios sistemas de regulación de la calidad de sus servicios.

La gestión académica es el ámbito operativo de interés para esta investigación, porque es a través de las acciones, decisiones y resultados académicos cotidianos que podemos evaluar la capacidad de las instituciones de responder con calidad a su doble exigencia. El énfasis está puesto en sondear las nuevas competencias adaptativas que deben desarrollar las unidades académicas, "...iqué tipos de mecanismos y medidas son aconsejables y eficaces para infundir flexibilidad al sistema académico y fortalecer su capacidad de cambio, adaptación e innovación con prontitud? ¿Cómo se puede mantener la estabilidad de un entorno que se encuentra en permanente cambio?"(Banco Mundial, 2003: 149).

Cuatro principios debieran sustentar la gestión académica de las UA:

- La pertinencia de sus planes de estudios e iniciativas académicas, de tal forma de asegurar a los alumnos y a sus futuros empleadores que el conocimiento y las competencias que caracterizarán su desempeño profesional responden a la excelencia académica en su disciplina.

- Debe asegurarse la suficiencia metodológica, es decir, incorporar las nuevas técnicas y medios, con el fin de facilitar las mejores condiciones de aprendizaje.

- Estos procesos, al igual que los administrativos, deben realizarse con máxima eficiencia, de tal forma de permitir al usuario concentrarse en el proceso pedagógico y no en procedimientos burocráticos que puedan desviar su concentración. 
- Se espera que las unidades académicas incorporen estrategias de innovación, accediendo con ello a las tecnologías y enfoques o medios que la ciencia va desarrollando y recomendando.

Es importante señalar que, con esto, no sólo se ofrecerá un buen servicio educativo a los alumnos, sino también se estará socializando una forma de pensar y de desempeñarse laboralmente en un contexto competitivo y riguroso.

Los procesos de gestión académica relevantes para el aseguramiento de la calidad de las carreras son variados. Para efectos de esta investigación, se han seleccionado los que parecen más urgentes de asegurar en una fase inicial de aprendizaje institucional para las carreras, a saber, planificación de procesos pedagógicos, monitoreo y seguimiento de resultados pedagógicos, evaluación, revisión y actualización curricular, vínculo con alumnos, ex alumnos, empleadores, recursos de aprendizaje, política de contratación de profesores, gestión de la información, del cambio, de RRHH y de resultados.

Fernando Lolas (2006) define una serie de desafíos para la gestión universitaria que, en este caso, aplicaremos a la gestión de las UA: formular metas a mediano y largo plazo, establecer prioridades para su cumplimiento efectivo, delegar adecuadamente el poder en autoridades legítimas, controlar el desarrollo de tareas, mantener la imagen pública, asegurar la viabilidad financiera y política, y proyectar la institución en el contexto social.

Cada uno de estos desafíos es un componente de la gestión universitaria y, por ende, debe llevarse a cabo integrando los dos planos en que ésta se desenvuelve. Por una parte el estratégico, es decir, lo definido en el proyecto educativo y el plan de desarrollo, y, por otra, el táctico u operacional, es decir, aquel que se desarrolla cotidianamente en las acciones, comunicaciones, decisiones y requerimientos del quehacer normal. La gestión académica debe entonces resguardar que los procesos estén enmarcados en directrices 
a largo plazo. A su vez, cada uno de ellos se instala en uno o varios procesos organizacionales, los mismos que analizamos al estudiar el desarrollo de una institución. Los procesos organizacionales de principal interés para esta investigación son la estructura, el liderazgo, la comunicación, la cultura y el clima. En conclusión, es a través de la optimización de los componentes de gestión instalados en los procesos organizacionales que aseguramos que los resultados de gestión académica cumplan con los cuatro criterios de excelencia previamente definidos. Lo que se pretende a través de esta investigación es indagar en qué medida las prácticas conducentes a la acreditación de las carreras inciden en dicha optimización.

\section{Referentes metodológicos}

\section{a) El problema}

Una vez instalada la preocupación por la calidad y su aseguramiento en los actores y las instituciones académicas, lo fundamental es conocer el espíritu y capacidad de AO de estas unidades. De igual manera, será posible saber si sus acciones propenden únicamente al cumplimiento de la tarea, con el fin de asegurar su integración al sistema oficial, o al fomento de una cultura de mejoramiento continuo en la gestión académica. Para sondear esto es necesario obtener información sobre los procesos de las unidades académicas; por ejemplo, el nivel de respaldo explícito por parte de las autoridades a las iniciativas de calidad, estructura en que se desarrollan los procesos, su validación y reconocimiento por parte de las UA y de la comunidad universitaria, nivel de recursos humanos y financieros destinados, metas, entre otros aspectos. Todo ello permite configurar un completo panorama respecto de la disposición institucional en la que se enmarcan los esfuerzos de cada carrera por la obtención de la acreditación. A través de esta información es posible conocer también cómo se está socializando la acreditación de carreras, qué tipo de información se está produciendo, en qué nivel y con qué tipo de procesos. 
Por otra parte, la posibilidad que ofrece el sistema de acreditar entre dos y siete años, según los niveles de calidad demostrados, se transforma en una amenaza para el efecto diferenciador que pretende incorporar el sistema con el sello de acreditación, ello porque la población no cuenta con un alto nivel de información técnica como para comprender el origen e implicancias de tal categorización. En lo concreto, es posible que, con este sistema, las carreras logren acreditarse sin maximizar sus prácticas de aprendizaje, desarrollo organizacional y autorregulación.

b) Objetivos de la investigación

Objetivo general:

Evaluar el impacto del proceso de autoevaluación en la gestión académica de algunas UA que se han sometido al proceso de acreditación de la CNAP en Chile.

Objetivos específicos:

1. Conocer, a través de un estudio de casos, la experiencia de implementación de procesos de autoevaluación de carreras en cinco universidades chilenas.

2. Conocer los principales cambios y transformaciones de los procesos de autoevaluación en el mejoramiento de la gestión académica de las UA.

3. Conocer prácticas y estrategias de gestión implementadas a partir de la decisión de autoevaluación, que luego se institucionalizan e instalan en la UA.

4. Contribuir a la generación de conocimientos respecto a los procesos de la educación superior chilena y sus sistemas de aseguramiento de calidad

c) hipótesis

1. La mayor parte de las carreras se han concentrado en instalar procesos, con el fin de cumplir con el procedimiento formal de acreditación, y no en hacer cambios sustanciales de gestión académica. 
2. Los resultados de acreditación (cantidad de años) de las UA no son necesariamente demostrativos de su capacidad e iniciativa para mejorar sus prácticas de gestión académica.

\section{d) Metodología}

Es un estudio de carácter cualitativo y exploratorio, ya que, como se ha señalado, su foco corresponde a una temática escasamente abordada como investigación en nuestro país y es necesario generar información para su análisis. Por esta razón, la primera etapa consistió en una entrevista a tres informantes clave asociados al sistema de acreditación, con el objetivo de orientar a la investigadora respecto del escenario y los puntos críticos del sistema. Las personas seleccionadas como informantes clave desempeñan un rol representativo del escenario universitario y pertenecen a alguna institución universitaria.

Se realizó un estudio de casos que contempló el trabajo con cinco universidades que habían implementado la acreditación de carreras obteniendo resultados diferentes entre ellas. Las instituciones están ubicadas en las ciudades de Valparaíso (V Región), Temuco (IX Región) y Santiago (Región Metropolitana-RM), con lo cual se buscaba dar cuenta de una selección heterogénea de casos.

Los criterios de selección de los casos que conforman la selección ${ }^{6}$ fueron:

- Tipo de institución: estatal o privada. Se seleccionó una universidad estatal de la RM y dos regionales, una universidad privada metropolitana creada en los primeros años de apertura del sistema a este sector y una segunda privada metropolitana creada posteriormente.

- Antigüedad: el hito definido para asignar las categorías antiguas o nuevas ha sido la apertura del sistema al mundo privado (1980).

6 Las universidades seleccionadas fueron: Universidad de Santiago (USACH), Universidad de Valparaíso (UV), Universidad de La Frontera (UFRO), Universidad Diego Portales (UDP), Universidad de Artes Ciencia y Comunicación (UNIACC). 
- Ubicación geográfica: regional o metropolitana

- Resultados en el proceso de acreditación ${ }^{7}$ : cantidad de años de acreditación de la carrera.

El liderazgo en el proceso de aseguramiento de la calidad al interior de una universidad compromete dos cargos fundamentales: el rector y el coordinador institucional de acreditación. Este último es una figura que recibe diversos nombres ${ }^{8}$, pero usualmente está instalado en la estructura superior, en un cargo transversal a todas las carreras. Si bien varían las funciones y cuotas de poder que se le asignan en cada institución, normalmente es quien está a cargo de coordinar y apoyar los procesos de acreditación de escuelas y de la propia organización. Por esto se decidió que éste sería la figura a través de la cual se presentaría la investigación a la universidad y, por tanto, la fuente primaria de información.

Las técnicas de recolección de información utilizadas fueron:

1. Entrevista en profundidad a las unidades de coordinación de acreditación y a las UA: en total se realizaron 15 entrevistas, cinco correspondiente a unidades coordinadoras y 10 correspondiente a carreras (ver pautas de las entrevistas en anexo).

2. Revisión de documentos institucionales, entre los cuales se encuentran los informes de autoevaluación, planes de desarrollo institucional, informes de otros procesos de evaluación, entre otros.

\section{Resultados, comentarios y sugerencias}

El análisis se estructurará sobre la base de tres componentes principales: procesos organizacionales, de gestión académica e impacto de la política en las unidades académicas y otros actores.

7 Los tres primeros criterios fueron utilizados para seleccionar las universidades, el último para seleccionar las carreras.

8 Por ejemplo, director de calidad, encargado de análisis institucional, coordinador de acreditación, etc. 
Estos componentes permiten poner énfasis en la capacidad de $\mathrm{AO}$ con que las unidades académicas enfrentan su proceso de acreditación.

\section{a) Componente organizacional}

Prácticamente todas las instituciones comenzaron espontáneamente a sondear el tema del aseguramiento de la calidad a mediados de los 90, ya fuese por un interés institucional o de algún funcionario. La gran mayoría debió enfrentar la implementación de esta política sin haber instalado en sus estructuras una instancia oficial especialmente abocada al tema, es decir, frente al requerimiento emergente, la mayoría de ellas tuvo que alterar su estructura organizacional. Esto se tradujo en la destinación de recursos financieros y humanos y la discusión directiva respecto de su dependencia y ubicación en el organigrama, lo cual fue un tema complejo, dado las características de las funciones que debían desempeñar, entre ellas, evaluación, monitoreo y análisis de información estratégica institucional, proceso de acreditación de pre y posgrado e institucional, por ejemplo.

“...inicialmente costó generarle un lugar a la oficina de autoevaluación y yo tenía discrepancias con las autoridades superiores; me querían dejar dependiendo de la vicerrectora académica, porque se suponía que los procesos de autoevaluación iban a los programas de pregrado". (Coordinador institucional).

Es posible apreciar que, entre las instituciones estudiadas, no hay un modelo único de unidad de aseguramiento de la calidad; algunas han delegado la tarea a unidades existentes (vicerrectoría académica o de desarrollo, por ejemplo), otras han fusionado esta tarea con la de análisis institucional, algunas han dedicado financiamiento especial, otras cuentan sólo con un encargado y otras con equipos multidisciplinarios. Lo que es común es que su configuración y dependencia ha sido producto de procesos no planificados en el diseño inicial de las instituciones. 
"La universidad partió con procesos experimentales de evaluación de la calidad de la docencia el año 97, a través de la creación de un programa. Éste dependía en sus inicios de la rectoría, dado que estas universidades tienen elecciones y obviamente entonces (...) hay distintos equipos que llegan a la rectoría, ha ido cambiando de alguna manera la ubicación jerárquica, si le quieres llamar así, organizacional del tema, pero no se ha dejado de trabajar en lo que es aseguramiento de la calidad si le quieres llamar así..." (Coordinador institucional).

Según los aportes de Rodríguez (2002), en mayor o menor medida, todas las universidades han demostrado capacidad de adaptación al entorno, "condición que por una parte asegura su supervivencia y que a su vez gatilla cambios internos" (Rodríguez, 2002: 220). Ello porque la falta de información sobre la acreditación al interior de las instituciones y la escasez de modelos procedimentales han sido características del proceso reconocidas por prácticamente todas las UA: todas ellas han debido improvisar, crear instrumentos, corregir acciones y dedicar tiempos y recursos especiales. La diferencia más importante se visualiza entre aquellas que aprovechan ese aprendizaje, lo extienden a otras unidades de la institución y lo utilizan para hacer más eficientes nuevos procesos v/s las que, luego de concluir el procedimiento, vuelven a su estado inicial.

La estrategia relacional usada por los coordinadores institucionales ha sido determinante en el éxito del proceso, debido a que, al comenzar los procesos de acreditación, el aseguramiento de la calidad era un tema desconocido, nuevo y escasamente debatido en las universidades. En la mayoría de las ocasiones las tareas asociadas a la acreditación fueron percibidas por académicos y administrativos como una carga extra de trabajo, más que como un proceso que facilitaría y optimizaría la gestión. Ha sido posible apreciar que, en la relación que los coordinadores institucionales establecen con las carreras, las siguientes características contribuyen al éxito de los resultados de las unidades coordinadoras: 
- Se definen ante las UA como organismos técnicos de apoyo para el procedimiento.

- Establecen una relación basada en el respeto por los tiempos y características de la carrera, sin imponerles tareas ni plazos.

- Cuentan con el respaldo explícito de las máximas autoridades de la universidad.

- Ofrecen apoyo técnico que contribuye a que las escuelas realicen un trabajo más eficiente (búsqueda de información, tabulación de datos, capacitación, entre otros).

Tal como señalan Silva, Ramírez y Atria (2002), si se trata de hacer un diseño o evaluación en una institución universitaria, es importante considerar que es un tipo de organización particular. Debe considerarse una adecuada flexibilidad con las subculturas propias de cada disciplina por las dificultades que podría ocasionar la tendencia del académico a imponer sus criterios profesionales. La flexibilidad de los procesos está asociada a la voluntad de los actores, pero también a las características de la estructura organizacional de la institución; es decir, a aquellas instituciones que se mantienen en un formato de administración tradicional y burocrático les es bastante más difícil establecer estrategias flexibles que consideren las particularidades disciplinarias de los cuerpos académicos o, incluso, las demandas de los estudiantes.

Un claro ejemplo son las complicaciones que han debido enfrentar algunas carreras respecto del lenguaje técnico o la metodología de trabajo del procedimiento de autoevaluación. La política está pensada para aplicar los procesos en forma homogénea en todas las carreras, generando resistencias por el desconocimiento de algunas disciplinas sobre estas materias. Lo más preocupante es que el efecto ha sido, en algunos casos, la resistencia al proceso. Por propia iniciativa y con recursos propios, las unidades académicas han tenido que suplir esta falencia del sistema y generar instancias de capacitación o socialización. En este contexto, es destacable que gran parte de las universidades en estudio hayan implementado diplomados relativos a los temas de gestión y calidad para los académicos y directivos de las escuelas. 
(Refiriéndose a la primera etapa del proceso de autoevaluación). "Bueno, lo primero fue organizarse, nosotros tomamos las propuestas que tenía el Ministerio para poder saber qué aspecto había que focalizar en esta autoevaluación, y lo que hicimos fue tener claridad. Tuvimos que tener un entrenamiento primero $y$, justamente, nos entrenaron a algunos de nosotros, vinimos a cursos para entender, había un lenguaje nuevo, en un tema que nosotros nunca habíamos tratado; por lo tanto, una vez aclarado todo eso, dijimos: ipor dónde vamos a partir, qué tenemos, qué no tenemos, cuáles van a ser nuestras limitaciones? Lo que sí teníamos como principio es que todo el mundo iba a tener que participar (...) porque o si no la gente no lo hace suyo". (Coordinador de carrera).

Por otra parte pudo apreciarse que las instituciones que desarrollaron estrategias concretas y exitosas de alineamiento en torno a un proyecto educativo común, cuyo objetivo es entregar formación de calidad, y que han concretado dichas intenciones en acciones incluso previas a los procesos de la CNAP, son aquellas que más fácilmente incorporan los procedimientos y se adaptan a las nuevas exigencias de la política.

(Refiriéndose a las dificultades de involucrar al equipo docente en el proceso de reacreditación) "Porque es más pega, es más trabajo, más reuniones y tú sabes que eso no te va a dar nada, que a lo mejor que aparezcas como participante de la comisión en el formulario: a ver, no es porque yo lo sea, pero la jefa de carrera tampoco recibe un peso extra". (Coordinadora de carrera).

Los procesos son exitosos en la medida en que la plana mayor de la institución se demuestre realmente comprometida con la acreditación, que incorpore el tema en el plan estratégico, que lo difunda en sus discursos, que propicie la asignación de recursos para la unidad, que otorgue autonomía al coordinador institucional. Estas 
medidas contribuyen a que la comunidad universitaria visualice esta meta como un esfuerzo común y tendiente al desarrollo institucional, haciendo posible en términos operativos su implementación. Si bien la gran mayoría de los equipos directivos de las universidades estudiadas habían definido la acreditación de las carreras como uno de los objetivos centrales de su gestión, esto no siempre se tradujo en acciones concretas. Tal es el caso de universidades en que la unidad de aseguramiento de calidad no cuenta con recursos propios o que tiene sólo un funcionario para cumplir con innumerables acciones.

(¿Y siempre hubo apoyo de parte del gobierno universitario?) "Lo que pasa es que formaba parte de las lógicas del gobierno (...) hubo un nivel de autonomía toda vez que en el discurso del rector estaba instalado ya el tema de la calidad, digamos, como herramienta de distinción institucional o una inspiración institucional a distinguirse por calidad, digamos, considerando que la universidad había decidido que no tenía la intención de desarrollarse en tamaño". (Coordinador institucional).

La designación del cargo de coordinador institucional es una decisión determinante en el éxito de los procesos, no sólo porque su nivel de autonomía depende habitualmente de su trayectoria y la confianza que los cargos directivos pueden depositar en él, sino también porque, dado lo novedoso del tema en el contexto universitario, la gestión de éste ha sido prácticamente una "evangelización", es decir, han tenido que gastar mucho de su tiempo en explicar el procedimiento y sus beneficios a los diversos actores del sistema. Asimismo, ha debido negociar con las autoridades de las escuelas y, especialmente, con quienes se oponen al proceso por temor a la estandarización de la educación. En este contexto, es sumamente importante que la persona que asuma esta tarea tenga condiciones de liderazgo, sea capaz de convocar al grupo de académicos y científicos a involucrarse en una tarea desconocida, con lenguaje desconocido y con una propuesta de evaluación normalmente muy diferente. 
Respecto a lo observado en las cinco instituciones, es posible afirmar que:

- El tamaño de la universidad es decisivo en los resultados del proceso de socialización del tema. En las universidades pequeñas la relación entre autoridades es más cercana y personalizada, son menos las carreras que hay que cubrir y, normalmente, las instancias colegiadas son más recurrentes, situación que facilita la comunicación entre el coordinador institucional y las autoridades de las carreras.

- Las características personales de los coordinadores influyen en su aceptación como líderes de un proceso complejo y demandante, desde el dominio sobre el tema hasta el nivel de confianza que establecen con los coordinadores de las carreras, todos son temas relevantes para el éxito de su estrategia.

- La autonomía que logren los coordinadores institucionales es determinante en los resultados. El coordinador puede ser desde sólo un "facilitador técnico", dependiente de las decisiones de vicerrectoría o de rectoría, hasta una autoridad autónoma en la institución, con atribuciones para manejar su propio presupuesto y crear instancias de apoyo al proceso. A diferencia de la característica anterior, ésta no depende del coordinador sino de la política institucional.

Tal como afirma Rodríguez (2001), actualmente se ha integrado el concepto de "liderazgo ejecutivo" a la teoría organizacional, ello porque esta capacidad puede ser un sustantivo aporte a los logros de una autoridad formal, en la medida en que logre reconocimiento y aceptación. Es decir, el desafío para quienes son asignados a las funciones de aseguramiento de la calidad en las instituciones y en las escuelas ha sido liderar los procesos de "evangelización" entre cuerpos docentes, equipos administrativos, organizaciones estudiantiles, entre otros estamentos.

En las universidades que se caracterizan por un clima cooperativo, de confianza y respeto los resultados han sido propicios. Ha sido muy valorado por las carreras que el coordinador institucional conozca a las personas que están a cargo del proceso de acreditación 
en cada escuela, que esté disponible para responder sus dudas y que facilite instrumentos $u$ orientaciones para el proceso. Las carreras demandan condiciones especiales para que sus docentes y administrativos puedan cumplir con las tareas asociadas a la acreditación. En las cinco universidades estudiadas se identificó éste como un tema crítico y si bien es cierto en algunas de ellas se ha avanzado, ninguna parece presentar las condiciones ideales en términos de horarios, incentivos y otros factores asociados.

"Nos gustaba mucho tomar decisiones en forma consensuada y que todos participaran, incluso yo diría que se da más de repente, uno invita a la gente y dice: 'no, mira, yo prefiero no estar en este comité. Oye, que se van a decidir cosas importantes. No, pero ustedes firmen'. O sea, es mi opinión, nunca hubo un problema que alguien dijera: '¡oh! me marginaron de la reunión, no me dieron información, no participé en eso'. Eso se ve muy poco". (Coordinador de carrera).

En lo que va del proceso, no se ha observado la generación de algún tipo de alianza entre universidades, ya sea convocada desde las mismas instituciones o desde el organismo central, que tenga como objetivo la socialización y transmisión de aprendizajes relevantes en la gestión de procesos de calidad. Aunque al interior de las universidades estudiadas existen algunas iniciativas que podrían presumirse como cooperación -la disponibilidad de la información institucional sistematizada por las primeras unidades acreditadas-, es posible afirmar que no existe una intención explícita de generar alianzas ni de traspasar el aprendizaje logrado por la unidad, sino que constituyen iniciativas aisladas y tendientes a la eficiencia.

\section{b) Componente gestión académica}

Hasta ahora hemos hecho un análisis de los resultados en términos generales, principalmente referidos a los procesos institucionales respecto de la acreditación de carreras. A continuación, pasaremos a revisar cómo ha incidido el proceso de acreditación en las variables 
asociadas a la gestión académica. Para esto tomaremos la clasificación de Fernando Lolas (2006) desarrollada antes. Es posible concluir que son principalmente tres los procesos en que se observan cambios relevantes en la mayoría de las carreras estudiadas, estos son: monitoreo y seguimiento de resultados, revisión y actualización curricular y vínculo con alumnos, ex alumnos y empleadores.

La gran mayoría de los representantes de las carreras declaran que uno de los principales aprendizajes de los comités de autoevaluación fue haber tomado conciencia de la importancia de sistematizar la información, los procesos y resultados, y, asimismo, respaldarlos con evidencia con el fin de asegurar la continuidad de sus iniciativas. Reconocen que normalmente los resultados de la sistematización de información inciden en el mejoramiento de procesos de planificación, evaluación, contratación de profesores y otros.

"Mira, yo creo que hubo una dificultad que a la postre se transformó en un plus, que era organizar el material: nosotros teníamos mucho material, teníamos muchas carpetas, pero estas carpetas obedecían a un orden cronológico, no tenían una organización de acuerdo con temas, entonces nos ayudó para organizar el material y darnos cuenta de todo lo que habíamos hecho". (Coordinador de carrera).

Por otra parte, al tratar de clasificar a sus escuelas en las cuatro categorías de AO señaladas por la investigadora, la mayoría señaló encontrarse entre el nivel 2 ó 3, ello porque la sistematización de información se ha transformado en una práctica habitual en cada unidad académica, pero además porque, en varios de los casos, las observaciones de los pares evaluadores detonaron una reformulación de los planes académicos. Para ello, algunas escuelas designaron comités especiales y otras siguieron trabajando con los equipos constituidos para la autoevaluación.

"Yo diría que hay como una autorregulación, no te diría que es completa, fantástica, pero sí que está esa semilla 
ahí, y que es una semilla que está germinando, o sea, aunque yo no tuviera que acreditarme nunca más, igual hay cuestiones que estoy revisando permanentemente, o sea de hecho ahora". (Coordinador de carrera).

Y por último, tal vez con la mayor incidencia de todas las nuevas prácticas que arrojó la investigación respecto a la gestión de las carreras, tenemos el vínculo con ex alumnos y empleadores. Si bien las carreras debieron generar estos procesos para conseguir su aplicabilidad en el procedimiento de autoevaluación, prácticamente todas señalan aprendizajes y resultados positivos a partir de estas acciones: les ha permitido generar alianzas, obtener información sobre el medio laboral, acceder a plazas de prácticas laborales, conocer sus propios resultados a través de la ubicación laboral de sus ex alumnos e incluso, en algunos casos, contratar como profesores a ex alumnos destacados.

Aunque en reiteradas ocasiones las carreras se han encontrado con resultados positivos no esperados, la implementación permanente de medidas para asegurar dichos procedimientos responde más a su funcionalidad para las escuelas que a lo estimado por los criterios de acreditación como mínimo exigible. Ello se traduce en que muchas de las acciones recomendadas en los planes de mejoramiento no se implementan durante varios años después de recibido el dictamen de la CNAP, aun cuando los comités están conscientes de los procesos de reacreditación que deberán enfrentar.

"Una de las cosas de que nos dimos cuenta es que nosotros somos súper buenos para innovar y siempre estamos pensando cómo hacer mejor las cosas, pero nunca evaluamos lo que hacemos". (Coordinadora de carrera).

Se observa una de las principales debilidades de los procesos en las escuelas: en muchas de ellas, las comisiones dejaron de funcionar luego del dictamen, traspasando las tareas de los planes de mejoramiento a las direcciones de escuela o de departamento. 
Aun cuando estas últimas instancias pudieran demostrar un real interés por continuar avanzando en esta dirección, se constatan dos fenómenos preocupantes:

- El tiempo destinado al trabajo de un director de escuela o de departamento no es el mismo que el de un comité destinado especialmente, con lo cual se pone en riesgo la efectiva implementación de acciones de mejoramiento.

- El aprendizaje logrado por los comités de autoevaluación no siempre es traspasado a los directores de departamento o de escuela, y esto se traduce en una pérdida de recursos, como también en un posible cambio de los criterios de trabajo.

Uno de los factores que permiten explicar esta discontinuidad en los procesos consiste en que la mayoría de las unidades coordinadoras institucionales colaboran permanentemente con las carreras sólo mientras dura el proceso de acreditación, pero en el transcurso éste y la reacreditación la mayoría no establece estrategias de continuidad.

(Respecto del apoyo de la coordinación central entre ambas fases) "Yo no lo he visto (el apoyo), porque no ha sido requerido tampoco. Yo creo que la carrera lo único que quiere es cumplir con estos compromisos de mejora, que si no se acerca a la dirección, no van a recurrir a ella, son por lo general aspectos internos..." (Coordinadora de carrera).

A pesar de que la acreditación promueve la autonomía como principio de gestión de las UA al cambiar un modelo de fiscalización por uno de monitoreo, el desarrollo de la cultura de AR aún es incipiente y, producto de ello, la mayoría de las escuelas reciben su dictamen y luego pierden continuidad en las acciones de mejoramiento. Es fundamental que la política de acreditación contemple una estrategia de acompañamiento, al menos en las universidades con estructuras más propensas a este fenómeno, con el fin de evitar el vicio del sistema. 


\section{C) Componente impacto de la política}

Es posible generar un análisis basado en tres elementos: (a) la percepción de los actores sobre la acreditación como mecanismo regulatorio, (b) ciertos componentes del proceso como la figura de los pares evaluadores y (c) nociones de calidad instaladas en los actores del proceso.

En primer término, la mayoría de los entrevistados asume que la acreditación es un proceso que, tarde o temprano, debía llegar a las universidades e, incluso, que debía generar más aspectos positivos que negativos, porque viene a ordenar un ámbito muy descuidado en temas de calidad. Es prácticamente una opinión generalizada el que la acreditación no ha logrado los resultados esperados en tanto elemento discriminatorio en el mercado, es decir, en el caso de alumnos y postulantes. Según los coordinadores de carreras e institucionales, ambos grupos conocen el término y demuestran nociones básicas al respecto, pero no lo utilizan como elemento de análisis para la toma de decisiones. Pareciera ser que la acreditación es un proceso que aún no llega a los postulantes e, incluso, a los alumnos, generando cierta apatía cuando éstos son informados por las escuelas sobre sus resultados.

Respecto de la iniciativa por comenzar a desarrollar procesos de acreditación en las carreras, se observa que en la mayoría de las instituciones se respetó la autonomía de las escuelas. El procedimiento utilizado por los organismos centrales fue, normalmente, invitar a las escuelas y hablarles sobre los beneficios de la acreditación. Entre los factores motivantes reconocidos por las mismas coordinaciones de carrera aparece el reconocimiento social o la diferenciación con sus pares.

Sobre el componente de evaluación externa, es importante señalar que, en algunos casos, sobre todo en las carreras que cuentan con un número menor de escuelas en el país, los equipos de trabajo se conocen, tienen experiencias previas y conocen las diferencias de sus enfoques de formación y trabajo. Esto en ocasiones genera resistencias, 
porque se percibe un intento por parte de los pares evaluadores de imponer una visión disciplinaria distinta de la transmitida en el proyecto académico en estudio. En ocasiones se percibió cierta tendencia a anular el aporte de los informes de pares, ya que se percibía que los esfuerzos realizados por los comités de autoevaluación se veían truncados por este factor externo. Esta situación parece ser bastante decisiva en la disposición de las carreras para continuar en el proceso.

En cuanto a las nociones conceptuales sobre calidad, a través de la última pregunta de la entrevista en que se solicita a coordinadores de carrera y de universidades que la definan, es posible concluir lo siguiente:

- Al escuchar la pregunta, prácticamente todos reaccionaron de igual manera: mostrándose confundidos y afirmando que era un tema complejo.

- No se identifica una conceptualización homogénea sobre calidad, algunas se centran en la infraestructura, otras en el desarrollo de competencias, algunas asignan responsabilidad únicamente a los cuerpos académicos y otras a todos los actores del sistema. Tal vez un parámetro común es que la mayoría de las definiciones entregadas por los coordinadores aducen coherencia entre lo que se intenta enseñar y lo que los alumnos son capaces de realizar al egresar de la universidad.

- Es posible que esto incida en el análisis sobre la capacidad que han tenido las autoridades y académicos de traspasar el sentido de la acreditación a sus alumnos. Es difícil pensar que podría haber un impacto importante si ni los mismos coordinadores cuentan con una clara definición al respecto. Este tal vez sea uno de los principales desafíos de una segunda etapa de implementación de la política.

Pareciera que hay una desconexión entre los procesos que implementan las escuelas para "asegurar calidad" y su concepción sobre este término. Se puede apreciar una desvinculación entre la conceptualización de calidad y el desarrollo de las respuestas previas, ya que, en general, no surgieron ejemplos sobre los procesos descritos 
o alusión a los temas tratados en las preguntas anteriores. Si bien a los entrevistados les es fácil hablar sobre procesos y procedimientos, pareciera ser que el concepto que guía dichas acciones no está aún suficientemente arraigado en el discurso institucional.

Por último, y como oportunidad de desarrollo para la política, ha sido posible percibir que los procesos de autoevaluación de las carreras adoptan matices según sus características disciplinarias, es decir, existen ciertas competencias desarrolladas por la profesión que facilitan o dificultan el desarrollo del procedimiento. Llama la atención, entonces, que el organismo central no considere esto a través de instrumentos y procedimientos ad hoc para optimizar los procesos.

"Otro tema es que diferentes carreras tienen diferente... potencial para llegar de manera autónoma al proceso, yo no te podría hablar de una cultura única en diferentes facultades, incluso departamentos académicos, hay diferentes posturas..." (coordinadora institucional).

\section{Conclusiones y recomendaciones}

Se comprueba que gran parte de las carreras se ha concentrado en instalar procesos para cumplir con el procedimiento formal de acreditación y no en hacer cambios sustanciales en su gestión académica. Con todo, aunque la disposición inicial haya sido cumplir con el procedimiento, han comenzado a tomar conciencia de los beneficios de la autorregulación, con lo que, espontáneamente, las carreras han mantenido algunas de las nuevas prácticas adquiridas con ocasión de la acreditación.

No se pudo comprobar que las UA que implementaron procesos de acreditación enfocados en el resultado y no en el proceso fuesen las que obtuvieron menor cantidad de años de acreditación. Sin embargo, que algunas carreras se hayan enfocado especialmente en el resultado no significa que no hayan logrado instancias de AO y, con ello, mejorar su capacidad de AR, aunque, normalmente, dichas instancias no fuesen planificadas. 
Se comprueba que los resultados de acreditación (cantidad de años) de las unidades académicas no son necesariamente demostrativos de su capacidad e iniciativa para mejorar sus prácticas de gestión académica, porque esa característica da cuenta de diversos procesos que ni la acreditación ni otro sistema estandarizado alcanzan a medir, como por ejemplo, procesos no contenidos en los criterios de acreditación.

La implementación de la Ley 20.129 de aseguramiento de la calidad marca un hito en los procesos de acreditación de la educación superior. Hasta ahora, los esfuerzos han estado explícitamente dirigidos a socializar la importancia de la calidad y de su certificación, y a involucrar a los actores en una nueva cultura. Por tanto, las recomendaciones que se desprendan de esta investigación son oportunas en tanto el organismo central, las agencias, las IES, alumnos, entre otros, tienen una nueva oportunidad ad portas: el comienzo de la segunda etapa del proceso de regulación de la calidad de la educación universitaria.

El tema de la calidad ha tenido un importante impacto en el sistema, talvez no aún en los estudiantes y postulantes -quizás porque no cuentan con el lenguaje ni los conocimientos técnicos como para entender una materia bastante compleja como ésta-, pero sí en la estructura pública, en las instituciones y en los poderes resolutivos del Estado. Como señala Navarro, las experiencias hasta ahora desarrolladas en este ámbito demuestran que las materias de acreditación han llegado a nuestra educación para quedarse y para constituirse en el pilar fundamental del aseguramiento de la calidad de la educación superior (Navarro, 2006). El éxito de la iniciativa es evidente y así lo reconocieron prácticamente todas las personas entrevistadas en esta investigación.

Tal como hemos señalado, este momento es crucial para el perfeccionamiento del proceso. El requerimiento más urgente es pasar desde una lógica de procedimiento a una de proceso. Hasta ahora se ha visto que, en la mayoría de los casos, el organismo central exige y las 
unidades académicas responden desde una lógica de cumplimiento. A pesar de que ello implica el desarrollo de nuevas competencias, no asegura el $\mathrm{AO}$ ni el mejoramiento continuo de las prácticas de gestión y su consecuente impacto en el mejoramiento de la calidad de la educación impartida.

La lógica de procedimiento está asociada a los sistemas de aseguramiento y al rol de fiscalizador que debe asumir el organismo central, en este caso la CNAP y las agencias. En cambio, la lógica de proceso se asocia con los sistemas de mejoramiento, los cuales, si bien es cierto implican mayor cantidad de recursos, aseguran el arraigo de una cultura de mejoramiento continuo, ya que se basan en un aprendizaje permanente. En palabras de Navarro, "hemos aprendido que, si bien es cierto hemos avanzado mucho en los procesos de aseguramiento de la calidad, es mucho más lo que queda por aprender y precisar. Por de pronto, tenemos que poner en cuestión el propio concepto de 'aseguramiento', que más propiamente debiera ser reemplazado por el de 'mejoramiento' puesto que, como lo señala Norman Sharp (2005), no basta con dar garantías sobre la calidad de los programas o de la gestión institucional, como lo sugiere el primero de estos conceptos, sino que además estos procesos deben estar arraigados en la naturaleza misma del aprendizaje efectivo, deben convertirse en parte de su cultura" (Navarro, 2006:122).

Si pudiéramos visualizar a los actores institucionales del sistema, es decir, al organismo central, las agencias y las IES como una gran organización, no hay hasta ahora señales de AO. No se observan medidas concretas que demuestren la capacidad de traspasar el aprendizaje, ni siquiera de sistematizarlo. Luego de ocho años de implementación del sistema hay información suficiente respecto de temas que permitieran modelar procesos y roles, como el de los pares evaluadores, las coordinaciones institucionales o los comités de AE; asimismo, información que permitiera conocer buenas prácticas de AE. Sin embargo, estos antecedentes no están fácilmente disponibles para la generalidad de los practicantes de la acreditación en las IES. 
Respecto del ámbito comunicacional, se aprecia una falencia importante, a pesar de que la generación de información, como instrumento discriminatorio para la toma de decisiones, es un pilar fundamental para el éxito de la acreditación. El impacto de ésta no ha alcanzado a llegar a los usuarios del sistema. En este sentido, podemos concluir dos cosas: por una parte, que las carreras no han logrado involucrar como se esperaba a sus alumnos en los procesos de AE. Tal parece que aún no se ha instalado una cultura de la calidad y no se ha logrado traspasar el sentido y la relevancia de este término. Por otra parte, la política tampoco ha logrado socializar completamente el valor de la acreditación como herramienta de toma de decisiones entre los postulantes a la educación superior.

La acreditación es, por definición, una variable nominal y, en virtud de esto, permite discriminar: una institución está o no está acreditada. Los motivos para asignar diferentes rangos de tiempo de acreditación son técnicos y difíciles de transmitir a la ciudadanía, lo cual afecta la calidad de la información pública. El usuario sólo sabe si la carrera está o no está acreditada, ya sea porque así lo informa la misma unidad o porque no distingue la relevancia de la segunda parte de la información.

\section{Sugerencias a la CNAP}

Considerando que la nueva función que cumplirá la agencia estatal es de carácter descentralizado, es decir, delegará funciones en nuevas agencias privadas, se sugiere que asuma una nueva función con énfasis en el mejoramiento del proceso. Para ello, proponemos acciones como:

- Difusión de buenas prácticas para optimizar los procesos de las universidades, por ejemplo, modelos de oficina de aseguramiento de la calidad o modelos de roles para los actores del proceso.

- Facilitar equipos formales de apoyo a las carreras, destinados específicamente a acompañarlas en la implementación de sus planes de mejoramiento; por ejemplo, en cambios curriculares, perfiles docentes y otros, en que normalmente los académicos no tienen dominio técnico sino más bien disciplinar. 
- Formación de instancias colaborativas entre IES, tendientes a fomentar el intercambio de aprendizaje organizacional y las alianzas estratégicas en esta materia.

- Formación y capacitación en temas transversales asociados a la acreditación; liderazgo de procesos de aseguramiento de calidad; implementación de sistemas de información; posibilidades de análisis, entre otras.

Las diferencias metodológicas entre disciplinas son una oportunidad que, al parecer, la política no ha aprovechado. Se sugiere hacer un diagnóstico de las competencias asociadas a cada disciplina en tanto inciden en las capacidades de AE y AR de sus programas docentes. Esta información permitirá hacer adaptaciones que pueden traducirse en un sistema más eficiente para las carreras, los coordinadores institucionales y las agencias.

Por otra parte, se recomienda a la CNAP y a las agencias acreditadoras incorporar un componente de acompañamiento a las carreras, en el intervalo entre la acreditación y primera reacreditación. En caso de que, por financiamiento, no sea posible cubrir todo el espectro de unidades académicas, por lo menos asegurar el acompañamiento de aquellas carreras más críticas que logran entre dos y tres años de acreditación. Tal como sugiere Navarro, "la experiencia demuestra que cuando los procesos de licenciamiento y de acreditación se encuentran muy cercanos, dos o tres años el uno respecto del otro, las instituciones tienden a repetir los antecedentes y hasta las formas de interpretarlos que les sirvió para alcanzar la autonomía" (Navarro, 2006: 115). Es posible pensar que en el caso de la acreditación y la reacreditación pasa más o menos lo mismo, sobre todo si consideramos el agotamiento expresado por las comisiones de autoevaluación y la tendencia a implementar los cambios en los últimos meses antes del segundo proceso.

La figura de acompañamiento podría asociarse a un rol de asesoría, en que el objetivo es ofrecer una retroalimentación permanente a las carreras. Estas asesorías pueden aprovecharse como instancia de transferencia de buenas prácticas y modelos. Asimismo, 
el informe que este asesor entregue a la comisión para evaluar la reacreditación contribuirá a conocer la calidad del proceso y no solamente los resultados logrados.

La percepción de los resultados de los informes de los pares evaluadores es tal vez una de las debilidades más importantes del sistema. Si bien se ponen en juego factores subjetivos asociados a la percepción de los actores, sería importante establecer estrategias que permitieran reducir el flanco de dudas. Parece que los conocimientos y capacidades que debe tener un par evaluador han de estar asociados no sólo a la disciplina y a la formación pedagógica, sino que, también, deben ser capaces de evaluar coherencia de planes estratégicos y proyectos institucionales, y tener habilidades sociales superiores para reflejar respeto por un proyecto educativo del que, talvez en su rol como académico de otra institución, discrepe. La selección de pares evaluadores parece hacerse, hasta ahora, únicamente sobre la base de criterios de experticia, tal como señala un par evaluador: "hay un momento de invitación inicial, que es bastante aleatorio, o sea yo creo que la gente de la CNAP se mueve sobre la base de datos, contactos, conocimientos previos, sugerencia que le hace gente más cercana a ellos, o sea, el primer momento de selección de un par evaluador es bastante fortuito".

Es recomendable mediatizar los criterios de selección de los pares evaluadores y agregar competencias genéricas que permitan reducir la resistencia de las carreras, sobre todo en ámbitos disciplinarios pequeños. Asimismo, perfeccionar el sistema de monitoreo y capacitación de estos actores.

Se sugiere buscar una estrategia de apoyo que permita aprovechar el aprendizaje de los pares evaluadores. Por ejemplo, tal vez ellos podrían estar a la cabeza de las instancias de capacitación y modelamiento de los coordinadores de carreras e institucionales.

Finalmente, sabemos que es deber del organismo central asegurar un sistema de información eficiente, función que hasta 
ahora sólo se ha remitido a la publicación de datos. Si se espera que éstos sirvan a la población como un instrumento para la toma de decisiones, es fundamental que se entreguen procesados. El alto impacto que tienen los medios de comunicación, prácticamente único medio por el cual los usuarios acceden a información sobre el sistema en la actualidad, pone en riesgo la objetividad de los datos que arrojan los estudios y, en muchas ocasiones, se pueden apreciar interpretaciones antojadizas o parciales. Tal como sugiere Navarro, la función de información debería estar instalada en diversos actores: el Ministerio de Educación, las propias IES y entes públicos y privados de distinta naturaleza, pero vinculados al campo de las informaciones en educación (Navarro, 2006: 109). Si cada uno de ellos asegura la entrega de información procesada y a través de medios efectivos, se facilitaría al usuario la triangulación de datos y se propendería a una mejor toma de decisiones.

\section{Sugerencias a las instituciones de educación superior}

Parece que uno de los temas críticos en la optimización de los resultados de las universidades es que han debido desarrollar competencias después de haber instalado los procedimientos de aseguramiento de la calidad y talvez el efecto más grave es que la mayoría de ellas, sobre todo en el caso de las instituciones más grandes, no cuentan con un diagnóstico de los recursos de su propia institución para enfrentar procesos de cambio.

Se sugiere a las instituciones implementar el modelo de análisis propuesto por Rosabeth Kanter, Barry Stein y Tood Jick (1992), denominado por sus autores como "modelo de los tres grandes". Éste considera:

- Tres tipos de movimiento: el de la organización como un todo, en referencia al movimiento del entorno; el de las partes de la organización respecto de las otras durante el crecimiento, envejecimiento y ciclo de vida de la organización, y el que resulta de la lucha por el poder y el control entre individuos y grupos de la organización. 
- Tres formas de cambio: de identidad en la relación entre la organización y su entorno; de coordinación que se refieren al ordenamiento interno de las partes que conforman la organización, y en el control, referidos a la dimensión política, las coaliciones dominantes, los intereses que se imponen, entre otros.

- Tres roles de acción en el proceso de cambio: estrategia de cambio, preocupada de la vinculación entre la organización y su entorno, y de orientar el movimiento de la organización en ese entorno (generalmente altos ejecutivos); gestor y ejecutor del cambio, dedicado a su implementación y a la coordinación interna de las partes (normalmente mandos medios); receptor del cambio, personas que son afectadas por éste, pero que no tienen mucha posibilidad de influir sobre él (habitualmente, personas de los últimos escalones de la jerarquía) (Rodríguez, 2001:230).

Este modelo permitiría a las universidades y carreras analizar las condiciones para la transformación, las principales dificultades previsibles y establecer, a partir de ello, estrategias para enfrentarlas. Asimismo, permitiría identificar los principales recursos y oportunidades con lo cual se podrían optimizar los resultados.

Al cabo de ocho años de implementación del sistema, ya es posible observar modelos exitosos en varios aspectos asociados a la política. Es posible sugerir un modelo de características que normalmente aseguran mejores resultados a las oficinas de aseguramiento de la calidad. Aunque no es posible aspirar a un modelo único de oficina, dada la relevancia del contexto estratégico y estructural de cada institución, sí es posible sugerir el análisis de las siguientes variables:

- Unidades que concentren todas las funciones de análisis institucional y acreditación: esta decisión optimiza la eficiencia de los procesos y la rigurosidad de los resultados, y, asimismo, contribuye a evitar duplicidad de información.

- Unidades autónomas, con dependencia directa de la rectoría y con apoyo explícito de las máximas autoridades de la universidad: la transparencia y credibilidad de las oficinas depende, en ocasiones, 
de su dependencia y autonomía, por la percepción que tenga la comunidad universitaria al respecto y por el respaldo de las autoridades, sobre todo en situaciones críticas o en toma de decisiones.

- Unidades que cuentan con personal y recursos permanentes para colaborar con las carreras, tanto en el proceso de acreditación, como en la implementación de los planes de mejoramiento.

- Unidades con capacidad predictiva y capacitadas para diagnosticar la situación interna -recursos, oportunidades de cambio, posibles resistencias- y externa -benchmarking, amenazas del entorno, temas emergentes, por nombrar algunos factores.

Se ha observado que la estructura de las oficinas institucionales es habitualmente centralizada, y que, si bien existen comités de autoevaluación en cada escuela, esta figura ha surgido en la coyuntura y no cuenta con las condiciones necesarias para funcionar permanentemente. Se sugiere implementar un modelo descentralizado, es decir, que la oficina institucional cuente con representantes en cada escuela, que tengan horas destinadas a esta función e, incluso, según el financiamiento lo permita, personal administrativo de apoyo a sus labores, considerando que gran parte de las funciones que deben desarrollar son de carácter administrativo y no académico.

Este último punto aborda un tema detectado en prácticamente todas las instituciones entrevistadas para esta investigación: el requerimiento de condiciones administrativas para llevar a cabo los procesos de acreditación. La prioridad de este tema en el plan estratégico de las universidades debe necesariamente traducirse en aspectos operativos como la asignación de personal, la asignación de fondos para capacitación y de horas para las coordinaciones de escuelas, la adquisición de textos o materiales, la contratación de asesorías, entre otros. 


\section{Referencias bibliográficas}

ACAP. (2006) Estudio internacional sobre criterios e indicadores de calidad de las universidades. Granada: ACAP.

Armanet, Pilar. (2004) ¿Por qué asegurar calidad? Calidad en la Educación CSE, (21), pp. 107- 122.

Atria, Raúl. (2005) Diversidad, Regulación y Universidad Pública. Revista de Sociología Universidad de Chile, (19), pp. 71-85.

Banco Mundial. (2003) Construir Sociedades de Conocimiento: Nuevos Desafíos para la Educación Terciaria, Washington: Banco Mundial.

Bernasconi, Andrés. (2004) Tendencias de cambio en la acreditación de escuelas: los casos de EE.UU., Australia y British Columbia. Santiago de Chile: Universidad Andrés Bello, pp. 1-8

Bernasconi, Andrés y Rojas, Fernando. (2003) Informe sobre la Educación Superior en Chile, Santiago de Chile: Universidad Andrés Bello, p. 17.

French, Wendell y Bell, Cecil. (1996) Desarrollo organizacional. México DF: Prentice- Hall, pp. 27-30.

González, Luis Eduardo. (2005) El impacto del proceso de evaluación y acreditación en las universidades de América Latina. IESALC/ UNESCO, (298), pp. 10- 20.

Hoecht, Andreas. (2006) Quality assurance in UK higher education: Issues of trust, control, professional autonomy and accountability, Higher Education Portsmouth Business School, (51), pp. 541- 563.

IESALC. (2006) Informe sobre la educación superior en América Latina y el Caribe 2000- 2005. Caracas: IESALC.

Korniejczuk, Víctor Andrés. (2003) La acreditación de la educación superior presencial y a distancia en Estados Unidos y México, Revista de Educación a Distancia (7), p. 5.

Lemaitre María José. (2003) Aseguramiento de la calidad en la educación transnacional, CNAP. VIII Seminario internacional 2003. Cruzando fronteras: Nuevos desafíos para la educación superior, (7), pp. 164169.

Lemaitre, María José. (2004) Mecanismos de aseguramiento de la calidad: respuesta a los desafíos del cambio en la educación superior. Calidad en la Educación CSE, (21), pp. 107- 122. 
Lepeley, María Teresa. (2003) Gestión y calidad en educación, un modelo de evaluación. México DF: Editorial McGraw-Hill, pp. 67- 69.

Lolas, Fernando. (2006) Sobre modelos de gestión universitaria. Revista Calidad en la Educación CSE, (24), pp. 35- 46.

Mok Ka Ho. (2000) Impact of globalization: A study of quality assurance systems of higher education in Hong Kong and Singapore. Comparative Education Review, University of Chicago, (44), pp. 170- 174.

Navarro, Iván. (2006) Acreditación: su desarrollo e impacto en la gestión institucional, Akademeia, (6), pp 103- 124.

Rodríguez, Darío. (2001) Gestión organizacional. Cambio organizacional. Santiago de Chile: Ediciones Universidad Católica de Chile, pp. 215238.

Rodríguez, Darío. (1999) Diagnóstico organizacional. Santiago de Chile: Ediciones Universidad Católica de Chile, pp. 31-63,165-186.

Romero, J. (2005) Introducción a la regulación. Santiago de Chile: Universidad Alberto Hurtado, pp. 6-7.

Senge, Peter. (2000) La danza del cambio. Los retos de sostener el impulso en organizaciones abiertas al aprendizaje. Colombia: Editorial Norma S.A.

Silva, Moisés y Letelier, Mario. (2003) Evaluación y acreditación en la universidad chilena. Evolución en perspectiva. Estudios Sociales (112), pp. 48- 50 .

Silva, Moisés; Ramírez, Soledad y Atria, Raúl. (2002) El proceso de autoevaluación y la universidad como organización. Estudios Sociales, (109), pp. 27-36.

Recibido: 3 de mayo de 2007

Aprobado: 28 de mayo de 2007 